\title{
Aplicações clínicas da hidrodinâmica na derivação ventrículo-peritoneal
}

\author{
Sebastião Gusmão, Roberto Leal Silveira, Guilherme Cabral Filho, Aluízio Arantes \\ Serviço de Neurocirurgia do Hospital Madre Teresa, Belo Horizonte, MG \\ Serviço de Neurocirurgia do Hospital Luxemburgo, Belo Horizonte, MG
}

\section{RESUMO}

A derivação ventrículo-peritoneal (DVP) é a aplicação do princípio hidrodinâmico dos vasos comunicantes. São freqüentes as complicações hidrodinâmicas em pacientes submetidos à DVP. Revisam-se os fatores hidrodinâmicos da DVP e suas complicações decorrentes.

\section{PALAVRAS-CHAVE}

Hidrocefalia. Derivação ventrículo-peritoneal.

\section{ABSTRACT their hydrodynamic complications were analyzed. \\ KEYWORDS \\ Hydrocephalus. Ventriculoperitoneal shunt.}

Clinical applications of hydrodinamics in ventriculoperitoneal shunt The ventriculoperitoneal shunt is an application of the hydrodynamic principle of communicating vessels. There are frequent hydrodynamic complications in the patients submitted to shunting. A revision of the hydrodynamic factors involved in the ventriculoperitoneal shunt was made and

\section{Introdução}

A derivação ventrículo-peritoneal (DVP) é o procedimento mais usado para tratar a hidrocefalia. Ela é uma aplicação do princípio hidrodinâmico dos vasos comunicantes. Tem por objetivo drenar o excesso de líquor para reduzir a pressão intracraniana e o tamanho dos ventrículos.

Em grande número de casos, não se consegue esse objetivo, persistindo a dilatação ventricular ou ocorrendo redução exagerada dos ventrículos ${ }^{1}$. $\mathrm{O}$ advento da tomografia computadorizada revelou alta freqüência de complicações hidrodinâmicas por inadequação entre o débito da DVP e a produção de líquor.

O objetivo desta revisão é analisar os fatores hidrodinâmicos da DVP e suas complicações decorrentes.

\section{Hidrodinâmica aplicada à derivação ventrículo-peritoneal}

No tratamento da hidrocefalia por DVP, estabelece-se a comunicação entre os ventrículos e a cavidade abdominal, através de cateter, com válvula interposta. O débito do líquor drenado é determinado pela lei de Poiseuille, expressa pela equação:

$$
\mathrm{D}=\Delta \mathrm{P} . \mathrm{r} 4 / 8 \mathrm{~nL} \text {, }
$$

onde $\mathbf{D}$ é o débito, $\Delta \mathbf{P}$ (pressão diferencial) é a diferença de pressão entre as duas extremidades do sistema de drenagem, ré o raio do tubo, $\mathbf{n}$ é a viscosidade do fluido e $\mathbf{L}$ é a distância entre as duas cavidades (comprimento do cateter de drenagem). No caso da DVP, o comprimento do tubo, o raio deste e a viscosidade do líquor podem ser considerados como constantes. Esses três elementos determinam a resistência $(\mathbf{R})$ do sistema à passagem do líquido. Assim, o débito da drenagem pode ser considerado como diretamente proporcional à diferença de pressão entre as duas extremidades do sistema, e inversamente proporcional à resistência do mesmo à passagem de líquido, o que é expresso pela equação:

$$
\mathrm{D}=\Delta \mathrm{P} / \mathrm{R}
$$

A válvula interposta na derivação assegura passagem em sentido único. Ela funciona quando existe diferença de pressão entre a entrada e a saída. $\mathrm{O}$ mecanismo de controle de pressão (fenda no silicone ou mola calibrada) mantém o sistema de derivação impermeável à passagem de líquido até determinado valor de $\Delta \mathrm{P}$ (pressão de fechamento de válvula). Quando $\Delta \mathrm{P}$ excede a pressão de fecha- 
mento, a válvula se abre, permitindo a passagem do líquido.

A pressão diferencial que age na DVP depende das pressões das cavidades proximal (ventrículos) e distal (abdome), da altura que as separa e da pressão de fechamento da válvula.

Pode-se, então, escrever:

$$
\mathrm{D}=\frac{\Delta \mathrm{P}}{\mathrm{R}}=\frac{\mathrm{PIV}+\mathrm{L}-(\mathrm{PIA}+\mathrm{PF}),}{\mathrm{R}}
$$

onde PIV é a pressão intraventricular, PIA é a pressão intra-abdominal, PF é a pressão de fechamento da válvula e $\mathbf{L}$ é a altura entre o ventrículo lateral e a extremidade distal do cateter peritoneal.

Os sistemas de válvulas para tratamento de hidrocefalia possuem mecanismo constante para controle da resistência. $\mathrm{O} \Delta \mathrm{P}$ sofre alterações inevitáveis e intensas em condições fisiológicas (como, por exemplo, mudanças de postura), podendo ocorrer, em conseqüência, grande variação do débito de líquor drenado. Isso dificulta que se consiga o objetivo principal do controle da hidrocefalia, ou seja, equilíbrio relativamente constante entre a produção e a drenagem do líquor.

A capacidade de drenagem do sistema de derivação liquórica é função de sua resistência à passagem de líquor e não de sua pressão de fechamento. Esta é apenas um dos fatores determinantes do $\Delta \mathrm{P}$ através do sistema de drenagem $(\Delta \mathrm{P}=\mathrm{PIV}+\mathrm{L}-\mathrm{PIA}-\mathrm{PF})$. Assim, as especificações das válvulas devem incluir não somente a pressão de fechamento, que não reflete completamente as características de funcionamento do sistema de derivação, mas também a resistência, definida pelo gráfico pressão/débito do teste hidrodinâmico.

As válvulas existentes podem ser divididas em duas categorias: de baixa e alta resistência.

\section{Pressão diferencial através da derivação ventrículo-peritoneal}

Os fatores determinantes da pressão diferencial através da DVP são: PIV, PIA, pressão hidrostática (altura do cateter) e resistência da válvula.

A pressão do líquor, em decúbito horizontal, é a mesma nos ventrículos, na cisterna magna e no fundo de saco lombar; varia de $50 \mathrm{~mm}$ a $200 \mathrm{~mm}$ de água ${ }^{2,3}$. Quando o paciente se encontra ereto ou sentado, a PIV no forame de Monro é, em média, de $\mathbf{- 7 0} \mathrm{mm}$ de água, o que corresponde à distância entre o orifício de Monro e a cisterna magna, onde a pressão é próxima de zero ${ }^{4,5}$. A PIV média no lactente é de $45 \mathrm{~mm}$ de água.
O conteúdo abdominal comporta-se como uma coluna de água. Assim, a PIA, como a PIV, é uma pressão hidrostática, estando o ponto “0”, na posição ereta, localizado na extremidade do apêndice xifóide $^{6}$. Em um paciente com DVP, a PIA que age sobre o sistema é igual à distância entre o ponto " 0 " e a extremidade do cateter abdominal. $\mathrm{Na}$ posição ereta ou sentada, a PIA cresce progressivamente da parte superior (nível " 0 ") até o fundo do saco de Douglas, onde atinge $300 \mathrm{~mm}$ de água (o que equivale à distância entre o ponto " 0 " e o fundo de saco de Douglas). Para o indivíduo adulto em decúbito horizontal, a PIA é de $80 \mathrm{~mm}$ de água em todos os pontos da cavidade abdominal. No lactente, esse valor é de $40 \mathrm{~mm}^{7}$.

A altura do cateter, que provoca grande pressão hidrostática quando o paciente se encontra na posição sentada ou ereta, representa o principal fator na determinação do $\triangle \mathrm{P}$ através da DVP. Ela varia segundo a implantação do cateter distal, a altura e a posição do indivíduo.

\section{Cálculo da pressão diferencial e da pressão intraventricular no paciente com derivação ventrículo-peritoneal}

Conhecendo-se os fatores responsáveis pelo $\Delta \mathrm{P}$ na DVP, pode-se determinar, teoricamente, o $\Delta \mathrm{P}$ e a conseqüente modificação da PIV causada pela derivação. $\mathrm{O} \Delta \mathrm{P}$ em um paciente submetido à $\mathrm{DVP}$ é dado pela equação:

$$
\Delta \mathrm{P}=\mathrm{PIV}+\mathrm{L}(\mathrm{PIA}+\mathrm{PF})
$$

Após a implantação da DVP, as pressões tendem a equilibrar-se, por meio da drenagem do líquor, de tal sorte que o $\Delta \mathrm{P}$ tende para valor nulo $(\Delta \mathrm{P}=0)$ em uma posição determinada. Amudança de posição do paciente vai acompanhar-se de novo equilíbrio. Quando o $\Delta \mathrm{P}$ for nulo, a derivação deixará de drenar, estando equilibrada a pressão entre as cavidades ventricular e abdominal. Nesse momento, a PIV será:

$$
\mathrm{PIV}=\mathrm{PIA}+\mathrm{PF}-\mathrm{L}
$$

Conhecendo-se aproximadamente os valores dos diversos fatores que intervêm no $\Delta \mathrm{P}$, pode-se calcular seu valor na DVP a ser implantada e a PIV após o equilíbrio do sistema de drenagem $(\Delta \mathrm{P}=0)$.

Como exemplo para o cálculo do $\Delta \mathrm{P}$ e da alteração da PIV provocada pela drenagem, tomam-se dois casos teóricos (Quadro 1): lactente com hidrocefalia hipertensiva e adulto com hidrocefalia de pressão normal submetidos à DVP com válvula de $70 \mathrm{~mm}$ de pressão de fechamento (válvula de pressão média). 


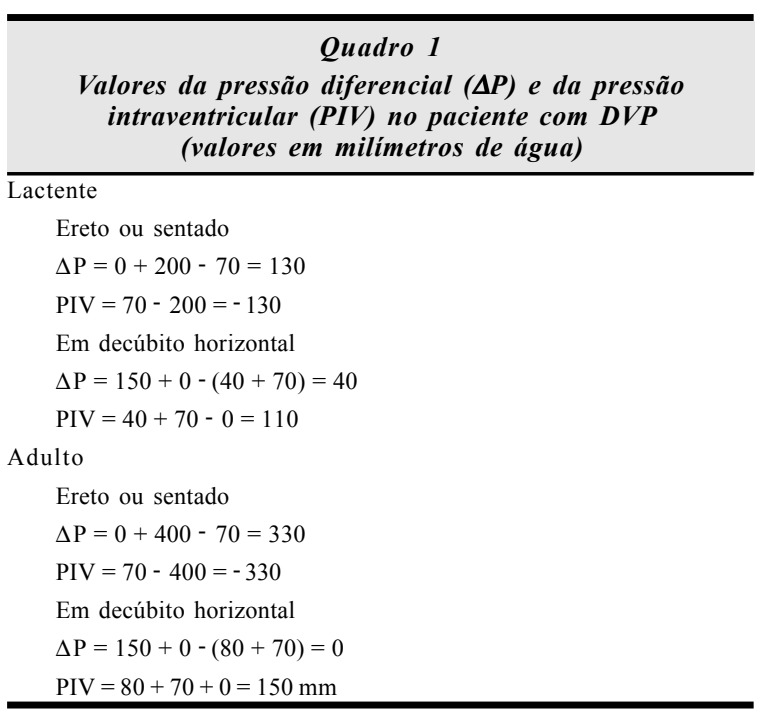

Nesse cálculo, usa-se o valor de $150 \mathrm{~mm}$ de água para a PIV na posição de decúbito, que é aproximadamente a pressão na hidrocefalia de pressão normal e também o valor médio da PIV na hidrocefalia da criança $^{8,9}$. Na posição ereta ou sentada, a PIV nos pacientes hidrocefálicos situa-se próximo de zero, podendo apresentar valores ligeiramente positivos ou negativos.

A PIA no paciente deitado é, aproximadamente, $40 \mathrm{~mm}$ de água no lactente e $80 \mathrm{~mm}$ de água no adulto ${ }^{6}$. $\mathrm{Na}$ posição ereta ou sentada, a PIA é anulada pela pressão hidrostática dentro do cateter, correspondente ao comprimento do cateter na cavidade abdominal. Assim, se tomarmos como altura do cateter (L) a distância entre a borda superior da órbita, que corresponde ao orifício de Monro, e o apêndice xifóide, não é necessário levar em consideração a PIA no paciente em posição ereta. Essa altura corresponde, em média, a $400 \mathrm{~mm}$ no adulto e a $200 \mathrm{~mm}$ no lactente.

Em decúbito horizontal, o $\Delta \mathrm{P}$ é pequeno ou nulo através da derivação e, conseqüentemente, ocorre pequena ou nenhuma alteração da PIV. Na posição ereta ou sentada, há grande aumento do $\Delta \mathrm{P}$, com significativa redução da PIV.

$O$ débito através da $D V P$ depende, além do $\Delta \mathrm{P}$, da resistência da válvula $(\mathrm{D}=\Delta \mathrm{P} / \mathrm{R})$. Assim, para um mesmo $\Delta \mathrm{P}$, uma válvula de alta resistência apresentará débito inferior ao de uma válvula de baixa resistência, reduzindo, em proporção menor, a PIV.

Mac Cullough e cols. ${ }^{5}$ e Fox e cols. ${ }^{10}$ mediram a PIV, na posição ereta, em pacientes adultos portadores de DVP, encontrando, respectivamente, os valores de $-320 \mathrm{~mm}$ e $-220 \mathrm{~mm}$ de água. Os valores calculados para a PIV (-330 $\mathrm{mm}$ de água) após derivação ventricular são bastante próximos dos valores medidos por esses autores.

\section{Complicações hidrodinâmicas}

As válvulas corrigem o volume de modo nãofisiológico, pois a produção do líquor é constante $(0,33 \mathrm{ml} / \mathrm{min})$ e o débito através da DVP varia segundo vários fatores.

Na posição ereta, a pressão hidrostática, determinada pelo comprimento do sistema de derivação (efeito sifão), leva a PIV a valores muito negativos, podendo determinar o aparecimento de complicações hidrodinâmicas por hiperdrenagem liquórica: síndrome de hipotensão liquórica, coleção subdural, alterações da forma e volume do crânio, desproporção craniencefálica, colapso ventricular e transformação de hidrocefalia comunicante em não-comunicante por estenose do aqueduto.

A síndrome de hipotensão intracraniana é a complicação mais freqüente da hiperdrenagem liquórica. Pode ocorrer acompanhada ou não de ventrículos em fenda (colapso ventricular). Manifesta-se por cefaléia, náusea e vômitos, diplopia, letargia, paresia do olhar conjugado para cima e estrabismo. Esses sinais e sintomas são semelhantes aos da hipertensão intracraniana por obstrução do sistema de derivação. Adiferença fundamental é que, quando se trata de hipotensão intracraniana, o quadro clínico manifesta-se quando o paciente se encontra em posição ereta e melhora quando assume a posição deitada. É fundamental a distinção entre as duas síndromes, pois o diagnóstico errôneo de hipertensão intracraniana por obstrução do sistema de derivação poderá levar à revisão cirúrgica desnecessária.

Foltz e Blanks ${ }^{4}$ descreveram 14 casos de hipotensão intracraniana em pacientes submetidos à DVP. Todos apresentavam a síndrome de hipotensão intracraniana, que era desencadeada pela posição ereta e melhorada pelo decúbito. Esses autores mediram a PIV desses casos, em posição ereta antes e depois da troca do sistema de derivação por outro de pressão mais elevada. A PIV média, no pré-operatório, era de $-136 \mathrm{~mm}$ de água e, no pós-operatório, de $-17 \mathrm{~mm}$ de água. Todos os pacientes apresentaram significativa melhora ou redução completa dos sinais e sintomas de hipotensão intracraniana após a substituição do sistema de drenagem por outro de mais eleveada pressão.

O quadro tomográfico de colapso ventricular é freqüentemente observado nos pacientes submetidos à derivação ventricular e é geralmente assintomático. Ocorre, com maior freqüência, em pacientes com mais de dois anos de idade e nos tratados com válvula de baixa resistência ${ }^{1}$. Decorre do maior $\Delta \mathrm{P}$ em pacientes com mais de dois anos de idade (posição ereta levando a alto valor da $\Delta \mathrm{P}$ ) e do maior débito nas válvulas de baixa resistência. 
O débito liquórico e a conseqüente alteração da PIV na posição ereta podem ser estimados pelo gráfico pressão/débito da válvula utilizada. Não existe, entretanto, solução simples para a hiperdrenagem liquórica, pois a derivação que funcione sob pressão diferencial que não determine hiperdrenagem na posição ereta pode provocar hipodrenagem na posição deitada.

Válvulas cujas resistências - e, conseqüentemente, a pressão de abertura - possam ser mudadas por meio de controle externo (válvula programável) foram desenvolvidas para adequar melhor o sistema de drenagem às necessidades do paciente, prevenindo, assim, as complicações hidrodinâmicas ${ }^{12,13}$. O mecanismo dessas válvulas baseia-se na variação da resistência exercida por uma mola conjugada a uma esfera, cujo movimento controla a abertura da válvula. A mudança de pressão é realizada por meio de ímã colocado sobre a válvula. Esse mecanismo elimina a necessidade de determinar a pressão de abertura ou a resistência ideal do sistema a ser implantado e a necessidade de revisão cirúrgica para mudar o sistema de derivação por outro de pressão ou resistência diferente.

Os casos de síndrome de hipotensão liquórica ou colapso ventricular, por hiperdrenagem liquórica, podem ser controlados por meio do simples aumento da pressão de abertura da válvula. Isso corresponde realmente ao aumento da resistência do sistema de drenagem pelo aumento da pressão sobre a mola que controla a abertura da válvula.

A complicação hidrodinâmica oposta à hiperdrenagem é a hipodrenagem liquórica. Trata-se de débito de drenagem liquórica inferior ao débito de produção do líquor, em derivação comprovadamente patente. Nesse caso, não ocorre melhora dos sinais e sintomas e a tomografia computadorizada evidencia persistência da dilatação ventricular. Tal eventualidade ocorre principalmente em lactentes e em pacientes tratados com válvulas de alta resistência'. Decorre do baixo valor do $\Delta \mathrm{P}$, por causa do pequeno comprimento do sistema de drenagem e da posição deitada do lactente na maior parte do dia, e do baixo débito nas válvulas de alta resistência.

A persistência da hidrocefalia por hipodrenagem liquórica é tratada com a substituição da válvula por outra de pressão de abertura menor ou de menor resistência. Quando se usa a válvula de pressão programável, basta alterar a pressão de abertura para valor menor, diminuindo, assim, a resistência da drenagem.

No pré-operatório pode-se avaliar, aproximadamente, o DP que vai agir na derivação e procurar indicar a válvula que melhor se adapte às necessidades do paciente. Assim, indica-se a válvula de alta resistência nos casos de alta DP (pacientes adultos com vida ativa) e a de baixa resistência nos casos de baixa DP (lactentes e pacientes acamados por longo tempo). Quando esses pacientes começarem a deambular, poderá ser necessário trocar a válvula de baixa por uma de alta resistência. A válvula de pressão programável simplifica a adequação da resistência da drenagem às necessidades do paciente.

A pressão intracraniana é constante fisiológica fundamental e sua alteração, para mais ou para menos, pode determinar lesões irreversíveis no sistema nervoso. A drenagem ventricular com resistência constante, funcionando por pressão diferencial, é uma forma grosseira de controle da pressão intracraniana. A válvula de resistência programável traz a vantagem de se poder controlar a resistência da drenagem, adaptando-se o débito de drenagem liquórica às necessidades do paciente.

\section{Referências}

1. AYER JB: Puncture of the cisterna magna. Arch Neurol Psychiatr 4:529-41, 1920.

2. DIETRICH U, LUMENTA C, SPRICK C, MAJEWSKI B: Subdural hematoma in a case of hydrocephalus and macrocrania. Experience with a pressure-adjustable valve. Childs Nerv Syst 3:242-4, 1987.

3. DUORNARCO JY, SOLOVEY G: Concepto de pressión intra-abdominal y estructura física del abdomen. Medicina 2:155-63,1942.

4. FOLTZ EL, BLANKS JP: Symptomatic low intracranial pressure in shunted hydrocephalus. J Neurosurg 68:401-8,1988.

5. FOX JL, Mac CULLOUGH DC, GREERT RC: Cerebrospinal fluid shunts: an experimental comparison of flow rates and pressure values in various commercial systems. J Neurosurg 37:700-5, 1972.

6. GUSMÃO S: Estudo da hidrodinâmica da drenagem ventricular e sua importância no tratamento da hidrocefalia. Tese (Mestrado). UFMG. Minas Gerais, 1984.

7. HAKIM CA: The physics and physicopathology of the hydraulic complex of the central nervous system. Tese (Doutorado). Massachusetts Institute of Technology. Boston, 1985.

8. HAYDEN PW, SHURTLEFF DB, FOLTZ EL: Ventricular fluid pressure recordings in hydrocephalic patients. Arch Neurol 23:147-65, 1970.

9. KONNO K, MEAD J: Static volume pressure characteristic of the rib cage and abdomen. J Applied Physiol 24:544-8, 1958.

10. LIGUORI G, CICCHIELLO L, AMBROSIO A, DANIELE $B$ : Continuous monitoring of intracranial pressure in non tumoral hydrocephalus of infancy. Acta Neurochir 38:143-51, 1977

11. LORNAN J: Components of cerebrospinal fluid pressure as affected by changes in posture. Arch Neurol Psychiatry 31:679-81, 1934. 
12. Mac CULLOUGH DC, FOX JL, CURL F, GREEN RC: Effect of CSF shunts on intracranial pressure and CSF dynamics. In Harbert TJC (ed): Cisternography and Hydrocephalus. Springfield, Thomas, 1972, pp 335-42.

13. Mac CULLOUGH DC, FOX JL: Negative intracranial pressure hydrocephalus in adults with shunts and its relationship to the production of subdural hematoma. J Neurosurg 40:372-5, 1974.
Original recebido em junho de 2000

Aceito para publicação em setembro de 2000

\section{Endereço para correspondência:}

Sebastião Gusmão

Rua Padre Rolim, 921 / 21

CEP 30130-090 - Belo Horizonte, $M G$ 